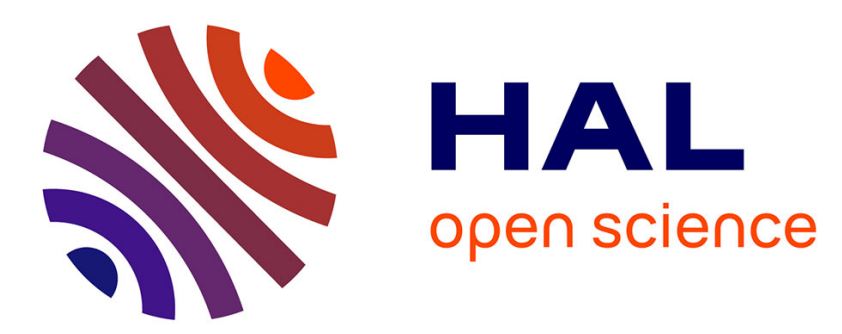

\title{
Energie élastique dans un matériau fritté subissant une transformation de phase
}

\author{
L. Eyraud, G. Malecot, Murielle Richard, R. Goutte
}

\section{To cite this version:}

L. Eyraud, G. Malecot, Murielle Richard, R. Goutte. Energie élastique dans un matériau fritté subissant une transformation de phase. Journal de Physique, 1963, 24 (4), pp.249-253. 10.1051/jphys:01963002404024900 . jpa-00205459

\section{HAL Id: jpa-00205459 https://hal.science/jpa-00205459}

Submitted on 1 Jan 1963

HAL is a multi-disciplinary open access archive for the deposit and dissemination of scientific research documents, whether they are published or not. The documents may come from teaching and research institutions in France or abroad, or from public or private research centers.
L'archive ouverte pluridisciplinaire HAL, est destinée au dépôt et à la diffusion de documents scientifiques de niveau recherche, publiés ou non, émanant des établissements d'enseignement et de recherche français ou étrangers, des laboratoires publics ou privés. 


\title{
ÉNERGIE ÉLASTIQUE DANS UN MATÉRIAU FRITTÉ SUBISSANT UNE TRANSFORMATION DE PHASE
}

\author{
Par L. EYRAUD, G. MALECOT, M. RICHARD, R. GOUTTE, \\ Faculté des Sciences de Lyon, Institut National des Sciences Appliquées de Lyon.
}

\begin{abstract}
Résumé - Toutes les fois qu'un échantillon polycristallin fritté subit une transformaton die phase au cours d'un refroidissement, et à condition que cette transformation soit parfaitement reversible, la variation anisotrope des paramètres cristallins crée dans le matériau des contraintes importantes. Au cours du refroidissement ce phénomène se traduit par une absorption d'énergie au milieu extérieur. Cette énergie est libérée brutalement par le matériau en cours de chauffage lorsqu'il passe au point de transition. Un calcul simplifié est effectué pour la transition cubique $\rightarrow$ tétragonale du titanate de baryum, souvent utilisé sous forme céramique. On voit en particulier l'influence de cette contribution énergétique à la chaleur totale de transition mesurée par microanalyse thermique différentielle.
\end{abstract}

\begin{abstract}
The anisotropic variation of the crystalline parameters of a sintered polycristalline material always creates important strains in the sample when the material undergoes a reversible change of phase. While cooling the sample, energy is absorbed by this process. This energy is quickly released when the material passes through the phase transition point by subsequent heating. A simplified calculation of the energy involved in the proposed process is described for the cubic tetragonal phase transition of barium titanate often used in the ceramic form. The results show in particular the magnitude of the elastic energy contribution to the total heat of transition measured by a thermal differential microanalysis technique.
\end{abstract}

Introduction. - Le titanate de baryum cristallise dans le système cubique au-dessus de $120^{\circ} \mathrm{C}$ (point de Curie). Entre $0^{\circ}$ et $120^{\circ} \mathrm{C}$ il est tétragonal et ferroélectrique. De nombreuses études expérimentales sur la thermicité de cette transition paraélectrique - ferroélectrique [1, 2, 3, 4, 5] n'ont pas encore permis de donner une explication valable du mécanisme de cette transformation solide-solide. Nos mesures, effectuées avec une cellule d'analyse thermique différentielle spécialement construite pour cette étude, conduisent à une variation d'enthalpie totale de

$$
\Delta H=0,21 \mathrm{c} / \mathrm{g} \pm 0,01 \text {. }
$$

Cette enthalpie correspond à une absorption d'énergie au milieu extérieur pendant la montée en température et au point de transition. On constate que cette énergie mesurée est toujours plus faible que la variation d'enthalpie calculée en ne tenant pas compte de l'énergie élastique.

Hypothèses de calcul. - Une céramique de titanate de baryum est un arrangement de grains répartis au hasard et collés entre eux. La cohésion est obtenue par compression puis chauffage à haute température $\left(1400^{\circ} \mathrm{C}\right.$ environ) dans la structure cubique. Aucune contrainte résiduelle de compression ne peut subsister au cours du refroidissement puisque l'échantillon a été porté à une température de ramollissement.

Cependant vers $120^{\circ} \mathrm{C}$ la transition cubiquetétragonale vient perturber l'arrangement cubique. Chaque cube élémentaire subit un allongement rela- tif $\Delta c / c=3,5 / 1000$ suivant une arête et un raccourcissement relatif $\Delta a / a=1,4 / 1000$ suivant les deux autres arêtes. L'hypothèse d'un réarrangement partiel est exclue par suite de la réversibilité presque parfaite de la transformation observée sur céramique. Il en résulte des contraintes internes intenses se traduisant par une absorption d'énergie au milieu extérieur.

Pour le calcul de cette énergie élastique absorbée par l'échantillon lors du passage cubique-tétragonal nous supposerons, pour simplifier, que la céramique est constituée de petits cubes, (comprenant une ou plusieurs cellules élémentaires) ayant chacun une face parallèle à un plan fixe pris pour plan de figure. Deux cubes voisins auront ainsi en commun tous les points d'une arête de l'un d'entre eux (fig. 1).

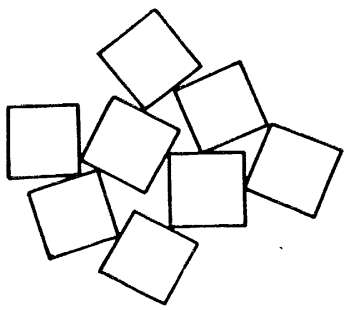

FIG. 1.

A) Calcul de l'énergie élastique. - 10 Supposons que l'anisotropie spontanée qui apparait au point de Curie avec la polarisation spontanée se 
produise dans le système cubique. Les relations entre contraintes et déformations s'écrivent :

avec

$$
\begin{aligned}
& T_{11}=c_{11} e_{11}+c_{12} e_{22}+c_{12} e_{33} \\
& e_{11}=\partial u / \partial x
\end{aligned}
$$

avec

$$
\begin{aligned}
& T_{22}=c_{12} e_{11}+c_{11} e_{22}+c_{12} e_{33} \\
& e_{22}=\partial v / \partial y
\end{aligned}
$$

avec

$$
\begin{aligned}
& T_{33}=c_{12} e_{11}+c_{12} e_{22}+c_{11} e_{33} \\
& e_{33}=\partial w / \partial z
\end{aligned}
$$

avec

$$
\begin{aligned}
& T_{23}=c_{44} e_{23} \\
& e_{23}=(\partial v / \partial z)+(\partial w / \partial y)
\end{aligned}
$$

avec

$$
\begin{aligned}
& T_{31}=c_{44} e_{31} \\
& e_{13}=(\partial u / \partial z)+(\partial w / \partial x)
\end{aligned}
$$

avec

$$
\begin{aligned}
& T_{12}=c_{44} e_{12} \\
& e_{12}=(\partial u / \partial y)+(\partial v / \partial x) .
\end{aligned}
$$

Les $T$ représentent les forces,

$$
e \text { les déformations, }
$$

$c_{\mathrm{ij}} \quad$ " les constantes élastiques de $\mathrm{Ba} \mathrm{Ti} \mathrm{O}_{3}$ dans le système cubique.

On a

$$
\begin{aligned}
& c_{11}=20,6 \times 10^{11} \mathrm{dyne} / \mathrm{cm}^{2} \\
& c_{44}=12,6 \times 10^{11} " \text { " } \\
& c_{12}=14,0 \times 10^{11}
\end{aligned}
$$

On peut admettre que sur chaque cube la composante normale $T=T_{11}=T_{22}=T_{33}$ et la composante tangentielle $T^{\prime}=T_{12}=T_{23}=T_{31}$ de chaque tension sont en moyenne égales; soit

$$
T=T^{\prime}=c_{44} e
$$

leur valeur commune.

$e$ représente la déformation angulaire moyenne ( $e=e_{23}=e_{31}=e_{12}$ ) d'un dièdre d'un cube.

Parallèlement à chaque arête de la figure, un cube subit une compression moyenne $e_{11}=e_{22}=e_{33}$ définie par la relation

$$
T=c_{11} e_{11}+c_{12}\left(e_{22}+e_{33}\right)
$$

soit encore

$$
e_{11}=\frac{T}{c_{11}+2 c_{12}}
$$

L'énergie élastique emmagasinée par unité de volume du matériau comprend : 1) trois travaux de compression de la forme $\left.(1 / 2) T e_{11} ; 2\right)$ trois travaux de couple de déformations angulaires de la forme $T e$.
I'énergie totale absorbée par unité de volume peut done s'écrire :

$$
U=T\left(\frac{3}{2} e_{11}+3 e\right)
$$

soit compte tenu de (1), (2) et des valeurs des $c_{\text {if }}$

$$
U \# 0,26 \times 10^{11}\left(c_{44} e\right)^{2}
$$

Calcul de la déformation angulaire moyenne e. Les déformations angulaires $e_{44}$ peuvent être calculées en considérant le triangle $\alpha \beta \gamma$ de la figure 2 formée par la coupe des arêtes de contact de trois cubes adjacents.

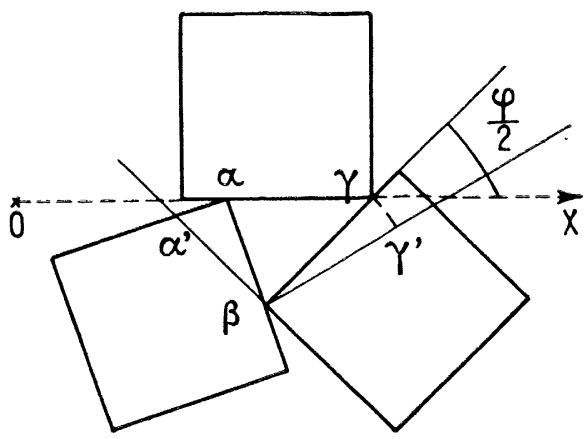

Fig. 2.

Les trois angles du triangle ont pour somme $\pi$. La valeur moyenne de chacun est donc $\pi / 3$. Nous prendrons $\widehat{\alpha \beta \gamma}=\pi / 3$. Comme le tiers seulement des arêtes croissent (4 sur 12), les autres étant pour le moment supposées constantes, nous calculerons la variation de cet angle quand les arêtes parallèles à la direction $\mathrm{O} x$ par exemple, croissent de $\Delta x=\Delta c=0,014 \AA$ suivant l'axe $c$.

Cette valeur $\Delta c=0,014 \AA$ a été obtenue par mesure aux rayons $X$ [7]. Nous étudierons plus loin le cas du raccourcissement $\Delta a=0,006$ suivant les deux autres arêtes lors du passage cubiquetétragonal.

Dans la déformation suivant l'axe $c, \alpha$ vient en $\alpha^{\prime}$ et $\gamma$ en $\gamma^{\prime}$ donc $\widehat{\alpha \beta \alpha^{\prime}}=\widehat{\gamma \beta \gamma^{\prime}}=\varphi / 2$ en moyenne (fig. 2).

Il revient au même de supposer $\alpha$ fixe et de prendre $\widehat{\gamma \beta \gamma^{\prime}}=\varphi$ pour faire le calcul de la déformation angulaire moyenne des triangles. Dans ce cas, on peut écrire $\alpha \gamma=c$ et $\alpha \gamma^{\prime}=c+\Delta c$.

On peut écrire dans le triangle $\gamma \alpha \gamma^{\prime}$ (fig. 3)

or

$$
\frac{\alpha \gamma}{\sin \frac{\pi}{6}}=\frac{\alpha \gamma^{\prime}}{\sin \left(\frac{\pi}{6}+\frac{\varphi}{2}\right)}=\frac{\gamma \gamma^{\prime}}{\sin \frac{\varphi}{2}}
$$

$$
\sin \left(\frac{\pi}{6}+\frac{\varphi}{2}\right) \# \frac{1}{2}+\frac{\varphi}{2} \frac{\sqrt{3}}{2}
$$


d'où

$$
\frac{\alpha \gamma^{\prime}}{\alpha \gamma}=\frac{c+\Delta c}{c}=1+\varphi \frac{\sqrt{3}}{2}
$$

soit

$$
\Delta c / c=\varphi \sqrt{3} / 2=0,014 / 4=3,5 / 1000 .
$$

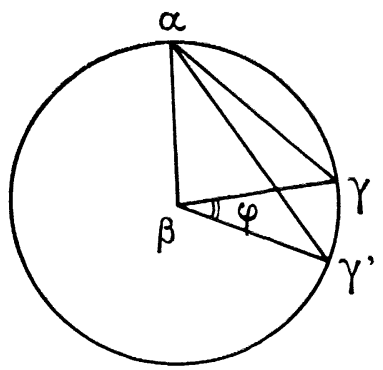

FIG. 3.

D'où la déformation angulaire moyenne de l'angle $\widehat{\alpha \beta \gamma}$ du triangle, correspondant à l'allongement suivant l'axe $c$

$$
\varphi=\frac{2}{\sqrt{3}} \frac{\Delta c}{c}=\frac{4}{1000}
$$

$\varphi$ est aussi la déformation angulaire d'un dièdre d'un cube lorsque les deux faces du dièdre tournent de $\varphi / 2$ en sens inverses, sinon la déformation est inférieure à $\varphi$. Il en résulte que $\varphi$ est une estima tion par excès de $e$.

Généralisation du calcul précédent à la transformation cubique-tétragonale. - La transformation ferroélectrique cubique-tétragonale considérée se traduit en fait par un allongement $\Delta c$ de la cellule élémentaire suivant un axe perpendiculaire aux faces mais aussi par un raccourcissement $\Delta a$ suivant les deux autres axes. On peut obtenir cette transformation géométrique par la succession des deux transformations suivantes :

$$
\begin{aligned}
& \text { système cubique } \quad a \quad a \quad c=a \\
& \text { affinité orthogonale fictive } \\
& \begin{array}{lll}
a & \downarrow & c
\end{array} \\
& \text { homothétie fictive }
\end{aligned}
$$

L'homothétie ne crée aucune distorsion dans le matériau cristallin. Seule l'affinité orthogonale, qui se traduit par l'allongement fictif $\Delta^{\prime} c$, produit une déformation angulaire

$$
\varphi^{\prime}=\frac{2}{\sqrt{3}} \frac{\Delta^{\prime} c}{c} \text {. }
$$

Or, d'après ce qui précède, l'allongement fictif $\Delta^{\prime} c$ est lié aux variations vraies des paramètres par la relation

$$
\begin{gathered}
c+\Delta c=\left(c+\Delta^{\prime} c\right)\left(1-\frac{\Delta a}{a}\right) \\
\Delta^{\prime} c \# \Delta c+\frac{c}{a} \Delta a \\
\Delta^{\prime} c \# \Delta c+\Delta a
\end{gathered}
$$

d'où la déformation angulaire moyenne d'un triangle

$$
\varphi^{\prime}=\frac{2}{\sqrt{3}} \frac{\Delta c+\Delta a}{c}
$$

La formule (4) nous permet alors de calculer l'énergie élastique par unité de volume dans le cas où l'on suppose l'anisotropie créée dans le système cubique et où l'on prend $e=\varphi^{\prime}$.

On a

$$
U=0,26 \cdot 10^{-11}\left(c_{44} \varphi^{\prime}\right)^{2} \mathrm{ergs} / \mathrm{cm}^{3} .
$$

Or

$$
\varphi^{\prime}=\frac{2}{\sqrt{3}} \frac{\Delta c+\Delta a}{c}=\frac{5,8}{1000}
$$

soit

$$
U=1387.10^{5} \mathrm{ergs}
$$

soit

$$
\begin{gathered}
\frac{13,87}{4,18} \text { calories } / \mathrm{cm}^{3} \text { et comme } \rho-6 \mathrm{~g} / \mathrm{cm}^{3} \\
U=0,55 \text { calorie/gramme. }
\end{gathered}
$$

Cette valeur est certainement plus élevée que la valeur réelle pour deux raisons :

a) Lues contraintes produites par les déformations du système cubique subsistent d'une façon permanente dans le système tétragonal dont les constantes élastiques sont différentes.

$b$ ) La déformation angulaire moyenne $\varphi^{\prime}$ d'un triangle est une estimation par excès de la déformation angulaire moyenne $e$ d'un dièdre.

$2^{\circ}$ Reprenons le calcul précédent en supposant que les contraintes apparaissent cette fois dans le système tétragonal ce qui est plus proche de la réalité.

I.es six constantes élastiques du système tétragonal sont alors (en dyne $/ \mathrm{cm}^{2}$ ) [6].

$$
\begin{array}{ll}
c_{11}=27,5 \times 10^{11} & c_{33}=16,5 \times 10^{11} \\
c_{12}=17,9 \times 10^{11} & c_{44}=5,43 \times 10^{11} \\
c_{13}=15,1 \times 10^{11} & c_{66}=11,3 \times 10^{11} .
\end{array}
$$

En admettant que les déformations angulaires des triangles sont, jusqu'au voisinage immédiat de la surface libre, de l'ordre de

$$
\varphi^{\prime}=\frac{2}{\sqrt{3}} \frac{\Delta c+\Delta a}{c}=\frac{5.8}{1000}
$$


et qu'elles s'exercent en majeure partie sur les dièdres dont la déformation est la plus facile (c'està-dire sur ceux qui ont une face carrée et une face rectangle, $c_{44}$ étant inférieur à $c_{66}$ ) dièdres étant d'ailleurs deux fois plus nombreux, et que, en outre, toutes les composantes $T_{\text {ij }}$ des tensions par unité de surface sur les faces sont en moyenne égales en valeur absolue à une même valeur

$$
T=c_{44} e_{23} \# c_{44} \varphi^{\prime},
$$

(la petitesse de $c_{44}$ montre que ces dièdres particuliers des tétragones résisteront beaucoup inoins que ceux des cubes à des pressions sur leurs deux faces et par suite que les déformations angulaires $e$ de ces dièdres seront souvent voisines de $\varphi^{\prime}$ ), les six déformations linéaires et angulaires seront en moyenne définies par les relations :

$$
\begin{aligned}
& T=c_{11} e_{11}+c_{12} e_{22}+c_{13} e_{33} \\
& T=c_{12} e_{11}+c_{11} e_{22}+c_{13} e_{33} \\
& T=c_{13}\left(e_{11}+e_{22}\right)+c_{33} e_{33} \\
& T=c_{44} e_{23}=c_{44} e_{31}=c_{66} e_{12} .
\end{aligned}
$$

L'énergie emmagasinée par unité de volume dans la phase tétragonale s'écrit alors comme prócédemment

$$
U=\frac{T}{2}\left[e_{11}+e_{22}+e_{33}+2\left(e_{23}+e_{13}+e_{12}\right)\right]
$$

des relations précédentes on tire

$$
\left\{\begin{array}{l}
T=\frac{c_{11}+c_{12}}{2}\left(e_{11}+e_{22}\right)+c_{13} e_{33} \\
T=c_{13}\left(e_{11}+e_{22}\right)+c_{33} e_{33}
\end{array}\right.
$$

soit en remplaçant les $c_{\mathrm{ij}}$ par les valeurs numériques

$$
\left\{\begin{array}{l}
10^{-11} T=22,7\left(e_{11}+e_{22}\right)+15 e_{33} \\
10^{-11} T=15\left(e_{11}+e_{22}\right)+16,5 e_{33} .
\end{array}\right.
$$

On a done sensiblement

$$
e_{11}+e_{22}+e_{33} \# \frac{T}{16} 10-11 .
$$

D'autre part, on peut encore écrire (dernière relaLion du systèmie 8)

$$
\begin{aligned}
& 2\left(e_{23}+e_{13}+e_{12}\right)=2 T\left(\frac{2}{c_{44}}+\frac{1}{c_{66}}\right)=2 T 10^{-11} \frac{28}{60} \\
& 2\left(e_{23}+e_{13}+e_{12}\right) \# \frac{9,3}{10} T 10^{-11} .
\end{aligned}
$$$$
\text { soit }
$$

D'où

Soit

$$
U=\frac{T^{2}}{2} 10^{-11}\left[\frac{1}{16}+\frac{9,3}{10}\right] \# \frac{T^{2}}{2} 10^{-11} .
$$

$$
\begin{gathered}
U=\frac{T^{2}}{2}{ }^{1-11} \mathrm{ergs} / \mathrm{cm}^{3} \text { et comme } T=c_{44} \varphi^{\prime} \\
U=0,5 \times 10^{-11}\left(c_{44} \varphi^{\prime}\right)^{2} \mathrm{ergs} / \mathrm{cm}^{3}
\end{gathered}
$$

en remplaçant $c_{\mathbf{4 4}}$. $\varphi^{\prime}$ par leur valeur $U \# 0,20$ calorie/gramme.
On voit que cette énergie élastique calculée n'est n'est pas négligeable et va intervenir d'une manière importante dans les résultats expérimentaux.

B) Introduction de cette Énergie élastique CALCUL ÉE DANS L'ÉNERGIE MESURÉE EXPÉRIMENTALEMent. - Au cours d'une montée en température et lors du passage de l'échantillon de $\mathrm{Ba}$ Ti $\mathrm{O}_{3}$ céramique au point de transition $\left(120^{\circ} \mathrm{C}\right)$ différentes énergies entrent en jeu.

1) L'énergie correspondant à la chaleur latente de transformation. Celle-ci peut se calculer à partir de la formule de Clapeyron

$$
\Delta H_{1}=\frac{T}{J} \frac{\mathrm{d} p}{\mathrm{~d} T} \Delta V .
$$

Cette énergie $\Delta H_{1}$, due à la variation de volume, est négative (absorption) et vaut

$$
\Delta H_{1}=-0,16 \mathrm{c} / \mathrm{g}
$$

en prenant $\Delta V=0,0376 \AA^{\mathbf{3}}[7]$.

2) L'érnergie de dépolarisation $\Delta H_{2}$. Celle-ci est négative et peut se calculer à partir de la valeur de la polarisation spontanée $P_{\mathrm{s}}$. Si l'on admet [8] que l'on a

$$
18 \mu \mathrm{C} / \mathrm{cm}^{2}<P_{\mathrm{s}}<26 \mu \mathrm{C} / \mathrm{cm}^{2}
$$

$\Delta H_{2}$ est telle que :

$$
0,18 \mathrm{c} / \mathrm{g}<\left|\Delta H_{2}\right|<0,36 \mathrm{c} / \mathrm{g} .
$$

3) L'énergie élastique $U$ positive calculée précédemment.

4) Les autres contributions énergétiques sont négligeables dans le cas du $\mathrm{Ba} \mathrm{Ti} \mathrm{O}_{3}$ polycristallin (énergie des murs de domaines, énergie du champ dépolarisant...).

Le bilan énergétique global du passage de l'échantillon de $\mathrm{Ba} \mathrm{Ti} \mathrm{O} \mathrm{O}_{3}$ céramique au point de transition dans le sens tétragonal $\rightarrow$ cubique (montée de température) donne donc comme énergie mesurable en analyse thermique différentielle:

$$
\Delta H=\Delta H_{1}+\Delta H_{2}+U
$$

soit avec les valeurs calculées précédentes

$$
0,14 \mathrm{c} / \mathrm{g}<|\Delta H|<0,32 \mathrm{c} / \mathrm{g} .
$$

la valeur trouvée expérimentalement

$$
\Delta H=-0,21 \mathrm{c} / \mathrm{g}
$$

sur des céramiques préparées par frittage à $1450^{\circ} \mathrm{C}$ pendant une heure s'inclue donc bien à l'intérieur des limites précédentes.

Conclusion. - Le calcul précédent donne une estimation de l'énergie de contrainte dans un matériau fritté subissant une transformation de phase. Cette contribution énergétique existe aussi dans les monocristaux ferroélectriques polydomaines, cristaux suffisamment gros pour que l'on 
puisse considérer une répartition aléatoire des directions de polarisation spontanée. Dans le cas particulier du titanate de baryum, ce calcul statistique simplifié montre pourquoi la variation d'enthalpie mesurée représente sensiblement l'énergie de pola- risation de la phase ferroélectrique. La chaleur latente de transformation $\Delta H_{1}$ étant compensée par l'énergie élastique de contraintes.

Manuscrit reçu le 27 octobre 1962.

\section{BIBLIOGRAPHIE}

[1] Blattner (H.) et Merz (W.), Helo. Phys. Acta, 1948, 21, 210.

2] Merz (W.), Phys. Rev., 1949, 76, 1221.

[3] Vul (B.), J. Physics U. R. S. S., 1946, 10, 95-105.

[4] Blattner (H.), Kanzig (W.) et Merz (W.), Hels. Phys. Acta, 1949, 22, 34-35.
[5] Volger (J.), Philips Research Reports, 1959, 7, 21-27.

[6] Huntington (H. B.), Solid State Physics, Acad. Press, New York, 1957, vol. 7, p. 280.

[7] Richard (M.), Goutte (R.) et Eyraud (L.), Résultats non publiés.

8] Merz (W.), Phys. Rev., 1953, 91, 513-517. 